\title{
Authentication analysis of red fruit (Pandanus Conoideus Lam) oil using FTIR spectroscopy in combination with chemometrics.
}

\begin{abstract}
Introduction: Red fruit (Pandanus conoideus Lam) is endemic plant of Papua, Indonesia and Papua New Guinea. The price of its oil (red fruit oil, RFO) is 10-15 times higher than that of common vegetable oils; consequently, RFO is subjected to adulteration with lower price oils. Among common vegetable oils, canola oil $(\mathrm{CaO})$ and rice bran oil (RBO) have similar fatty acid profiles to RFO as indicated by the score plot of principal component analysis; therefore, $\mathrm{CaO}$ and $\mathrm{RBO}$ are potential adulterants in RFO. Objective: To develop FTIR spectroscopy in combination with chemometrics of partial least square regression (PLSR) and discriminant analysis (DA) for authentication of RFO from $\mathrm{CaO}$ and $\mathrm{RBO}$. Results: The presence of $\mathrm{CaO}$ in RFO was better determined at frequency regions of $1200-1050 \mathrm{~cm}^{-1}$; meanwhile, the combined frequency ranges of 1207-1078 and 1747-1600 $\mathrm{cm}^{-1}$ were exploited for quantitative analysis of RBO with acceptable values of coefficient of determination $\left(\mathrm{R}^{2}\right)$ and errors in calibration, prediction and during cross validation. DA based on Mahalanobis distance was able to discriminate between RFO and RFO adulterated with $\mathrm{CaO}$ and RBO. Conclusion: FTIR spectroscopy combined with PLSR and DA can be successfully used for quantification and classification of oil adulterants in RFO. The developed method is rapid and environmentally friendly and sample preparation is easy.
\end{abstract}

Keyword: FTIR spectroscopy; Partial least square regression; Discriminant analysis; Authentication. 